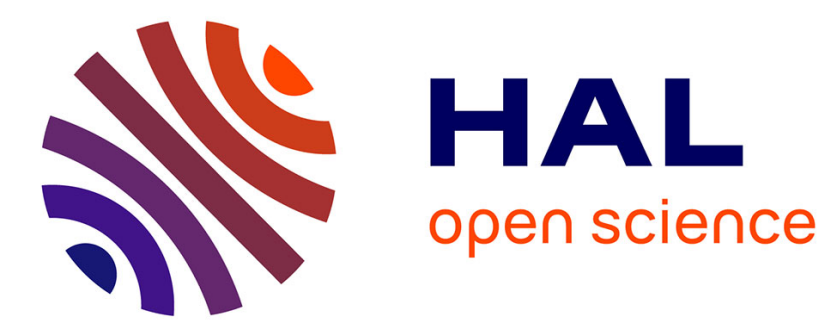

\title{
The Optimality of PFPasap Algorithm for Fixed-Priority Energy-Harvesting Real-Time Systems
}

\author{
Yasmina Abdeddaïm, Younès Chandarli, Damien Masson
}

\section{To cite this version:}

Yasmina Abdeddaïm, Younès Chandarli, Damien Masson. The Optimality of PFPasap Algorithm for Fixed-Priority Energy-Harvesting Real-Time Systems. ECRTS 2013, Jul 2013, France. pp.47-56. hal-00783607v2

\section{HAL Id: hal-00783607 https://hal.science/hal-00783607v2}

Submitted on 29 Apr 2013

HAL is a multi-disciplinary open access archive for the deposit and dissemination of scientific research documents, whether they are published or not. The documents may come from teaching and research institutions in France or abroad, or from public or private research centers.
L'archive ouverte pluridisciplinaire $\mathbf{H A L}$, est destinée au dépôt et à la diffusion de documents scientifiques de niveau recherche, publiés ou non, émanant des établissements d'enseignement et de recherche français ou étrangers, des laboratoires publics ou privés. 


\title{
The Optimality of $P F P_{A S A P}$ Algorithm for Fixed-Priority Energy-Harvesting Real-Time Systems
}

\author{
Yasmina Abdeddaïm, Younès Chandarli and Damien Masson \\ Université Paris-Est, Laboratoire d'Informatique Gaspard-Monge UMR 8049, \\ UPEMLV, ESIEE Paris, ENPC, CNRS, F-93162 Noisy-le-Grand France \\ y.abdeddaim@esiee.fr / younes.chandarli@univ-paris-est.fr / d.masson@esiee.fr
}

April 29, 2013

\begin{abstract}
The paper addresses the real-time fixed-priority scheduling problem for battery-powered embedded systems whose energy storage unit is replenished by an environmental energy source. In this context, a task may meet its deadline only if its cost of energy can be satisfied early enough. Hence, a scheduling policy for such a system should account for properties of the source of energy, capacity of the energy storage unit and tasks cost of energy. Classical fixed-priority schedulers are no more suitable for this model. Based on these motivations, we propose $P F P_{A S A P}$ an optimal scheduling algorithm that handles both energy and timing constraints. Furthermore, we state the worst case scenario for non concrete taskset: ${ }^{1}$ scheduled with this algorithm and build a necessary and sufficient feasibility condition for non concrete tasksets. Moreover, a minimal bound of the storage unit capacity that keeps a taskset schedulable with $P F P_{A S A P}$ is also proposed. Finally, we validate the proposed theory with large scale simulations and compare our algorithm with other existing ones.
\end{abstract}

\section{Introduction}

Due to the growing demand for smaller devices with longer battery life, energy management has become one of the major goals in embedded systems research. Indeed, a naive use of the energy available on board can lead to a short runtime for these devices. However, the targeted embedded applications can be required to operate over long periods after they are deployed, for example, in the case of sensor nodes. The extended life of these electronic devices is of particular importance when they have limited accessibility. Thus, collecting energy from the ambient environment can be a very interesting solution, which is known as Energy Harvesting. In this process, energy is drawn from the environment and then converted and stored for use in electronic applications. Compared to classical energy storage devices, the environment proves to be an infinite source of available energy. Furthermore, using this kind of energy eliminates the need to replace batteries periodically that constitute a major part of service and maintenance.

Many environmental sources can be exploited, including thermal, optical, mechanical, fluid, etc. Energy sources must be considered according to the characteristics of the application. Self powered sensors for medical implants and remote condition monitoring embedded sensors in structures such as bridges or buildings are typical examples of targeted applications.

In addition, the applications running on power-limited systems can be subject to timing constraints. Consequently, real-time and energy-aware features are both highly desirable and sometimes crucial for such systems. An energy harvesting system is composed of three parts:

The harvester is the part that converts the energy from ambient surroundings into usable electrical power.

\footnotetext{
${ }^{1}$ a non concrete taskset is a set of real-time tasks whose offsets are known only at run-time
} 
The storage unit is a device used to store the electrical energy produced by the harvester (e.g. a rechargeable battery or a capacitor).

The computing system is a real-time system that uses the energy stored in the battery to run the softwares.

In this paper we are interested in the problem of real-time scheduling for Energy-Harvesting systems. The challenge is to schedule real-time tasks and to make the best use of the available energy which is highly dependent on the environment. The energy consumption of the system should be adjusted to maximize its performance instead of minimizing its overall energy consumption as in classical battery-powered systems. A new role of the operating system is to properly manage the activity of the processing unit so that, at any time, there is sufficient energy in the storage unit to satisfy all the constraints.

This work focuses on optimal fixed-priority solution of this problem. The remaining part of the paper is organized as follow: first, we present the related work in Section 2, The model is described in Section 3. In Section 4 we introduce $P F P_{A S A P}$, a fixed-priority scheduling algorithm, and then we study some of its properties, namely the worst case scenario and its optimality for non concrete traffic. A feasibility condition based on PFPASAP is also proposed. In Section 5 we evaluate the performance of $P F P_{A S A P}$ and we compare it with other algorithms by performing large scale simulations. Finally, we discuss the themes of future work, then, we conclude in Section 6.

\section{Related Work}

Researchers started to address the issues of power and scheduling only in the past decade with the objective of either minimizing power usage under timing constraints or maximizing the system performance under energy constraints. Nevertheless, most of them have not considered the limited capacity of the battery and the need to manage its continuous replenishment.

Until recently, the most of this research has focused on saving energy using the DVFS technique (Dynamic Voltage and Frequency Scaling) [16, 15]. The idea here is to save energy by slowing down the processor just enough to meet tasks deadlines.

These techniques have limitations in energy harvesting systems because they increase the probability of transient faults [16, 15] and cannot be used alone in case where there is not enough energy to execute. An energy aware scheduling strategy for harvesting systems must dynamically manage tasks according to the profiles of both available energy and the workload of the processor.

The first work which addressed the scheduling problem of energy harvesting systems was presented by Mossé in [2]. The problem was solved under a very restrictive task model: the framebased model where all the tasks have exactly the same period and the same implicit deadline. Later in [14, Moser et al. proposed an optimal algorithm called $L S A$ (Lazy Scheduling algorithm) for periodic or aperiodic tasks. However, in their hypotheses, the CPU frequency can be changed to adjust the Worst Case Execution Time (WCET) of the tasks depending on their energy consumption. Thus, the results of this work rely on the assumption that task energy consumption is directly linked to their WCET. Recent work shows that this hypothesis is not suitable for embedded systems [1].

Later, a clairvoyant algorithm called EDeg and several heuristics have been proposed in [10, 7 . In this context, an algorithm is said to be clairvoyant if it takes scheduling decisions according to the processor and the energy load, the amount of incoming energy and the energy level in the storage unit. The algorithm EDeg relies on a generalizable meta policy: as long as the system can perform without energy failure, a standard policy such as EDF is used. Then, as soon as future energy failure is detected, the system is suspended as long as possible depending on timing constraints or until the energy storage unit is full. To detect such future energy failure, the notion of slack time [12] was extended to the notion of slack energy. This algorithm was evaluated with non clairvoyant heuristics that schedule jobs as soon as possible until energy runs out, then suspend the system a while without looking for the future state of energy. For example they suspend the system for a fixed period of time or consume all available slack-time to replenish the battery. 


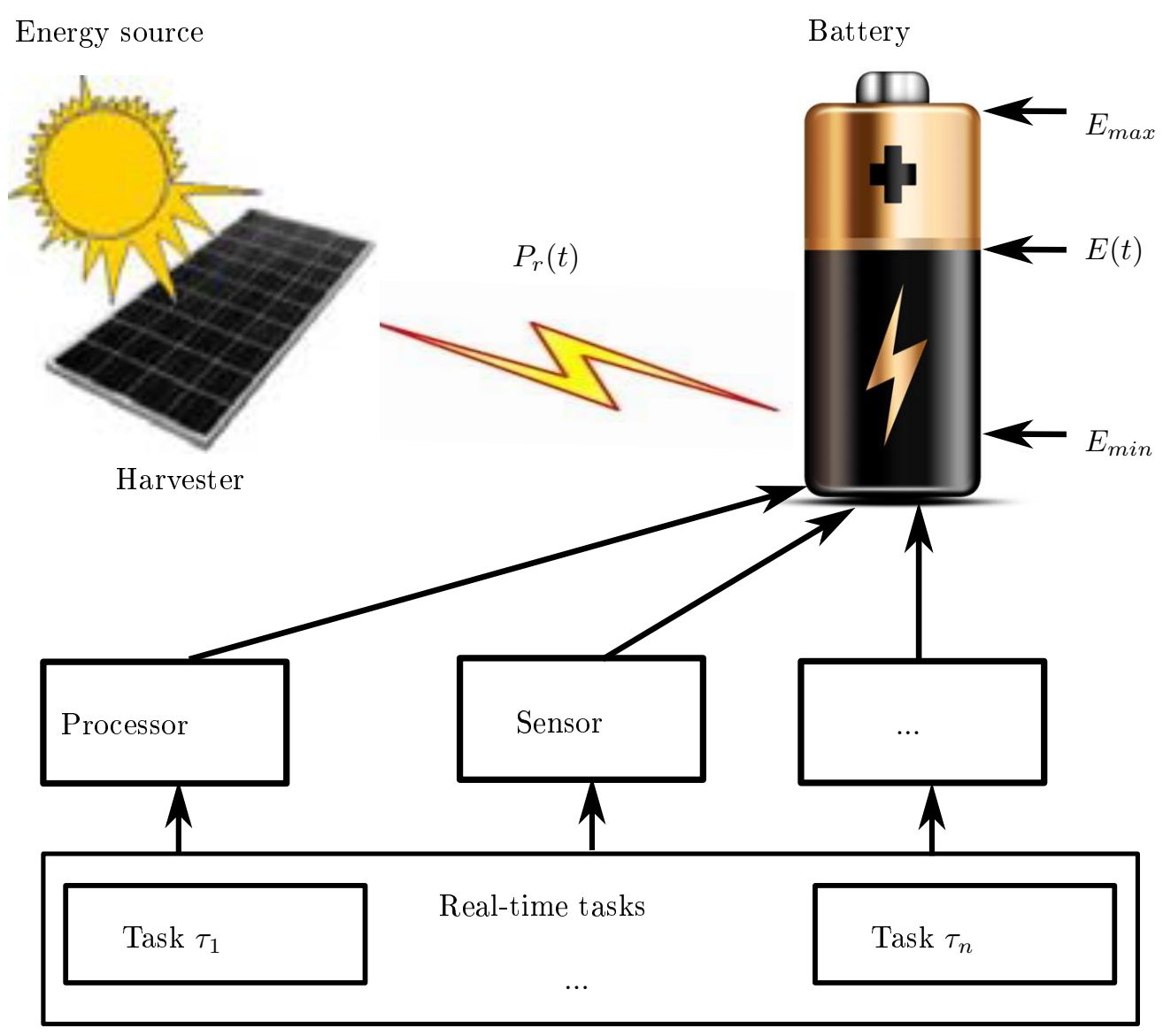

Figure 1: Energy Harvesting Embedded System Model

Most work about scheduling energy-harvesting systems focus on dynamic priority scheduling by proposing algorithms mainly based on $E D F$ because of its optimality for classical scheduling problems. However, most of embedded systems usually operate with fixed-priority scheduling policies because of their simplicity and their low overhead.

\section{Problem Statement}

\subsection{Taskset Model}

We consider a non concrete real-time taskset in a renewable energy environment defined by a set of $n$ periodic and independent tasks $\left\{\tau_{1}, \tau_{2}, \ldots, \tau_{n}\right\}$. Each task $\tau_{i}$ is characterized by its priority $P_{i}$, its worst case execution time $C_{i}$, its period $T_{i}$, its deadline $D_{i}$ and its worst case energy consumption $E_{i}$. The execution time $C_{i}$ and the energy consumption $E_{i}$ of a task are fully independent, for example considering two tasks $\tau_{i}$ and $\tau_{j}$, we can have $C_{i}<C_{j}$ and $E_{i}>E_{j}$. A task $\tau_{i}$ releases an infinite number of jobs separated by $T_{i}$ time units and each job must execute during $C_{i}$ time units and consume $E_{i}$ energy units. All the tasks consume energy linearly, i.e. a constant amount of energy for each execution time unit. Deadlines are constrained or implicit. The taskset is priority-ordered, task $\tau_{n}$ being the task with the lowest priority. Since the considered taskset is a non concrete one, the offsets denoted as $O_{i}$ are known only at runtime. 


\subsection{Target Application Description}

We consider an embedded system connected to an energy harvesting device. An energy harvesting device is a system collecting energy from its environment (e.g. with a solar panel). The collected energy is stored in an energy storage unit with fixed capacity (e.g. chemical battery or capacitor). We suppose that the quantity of energy that arrives in the storage unit is a function of time which is either known or bounded. As mentioned in Section 3.1 task energy and processor cost are fully independent. Indeed, in practice, a task can use some devices that are independent from the processor (e.g. sensors, engines). Even if we consider only the processor consumption, the later relies heavily on the kind of circuitry that is used by the code, rather than on the duration of its execution 11 .

The replenishment of the storage unit is performed continuously even during the execution of tasks, and the energy level of the battery fluctuates between two thresholds $E_{\min }$ and $E_{\max }$ where $E_{\max }$ is the maximum capacity of the storage unit and $E_{\min }$ is the minimum energy level that keeps the system running. The difference between these two thresholds is the part of the battery capacity dedicated to tasks execution, denoted as $\mathcal{C}$. We suppose that $\mathcal{C}$ is sufficient to execute at least one time unit of each task. This means that $\mathcal{C}$ must be greater or equal to the maximum instantaneous consumption, i.e. $\mathcal{C} \geq \max _{\forall i}\left(E_{i} / C_{i}\right)$, otherwise the taskset cannot be executed. For the sake of clarity, we can consider without loss of generality that $E_{\text {min }}=0$ and that $\mathcal{C}=E_{\max }$. The battery level at time $t$ is denoted as $E(t)$. As the tasks offsets, the initial level of the battery $E(0)$ is unknown before runtime. We note $P_{r}(t)$ the replenishment function of the battery, then, the energy replenished during any time interval $\left[t_{1}, t_{2}\right]$ denoted as $g\left(t_{1}, t_{2}\right)$ is given by Formula 1 .

$$
g\left(t_{1}, t_{2}\right)=\int_{t_{1}}^{t_{2}} P_{r}(t) d t
$$

To simplify the problem, we assume $P_{r}(t)$ to be a constant function, i.e. $P_{r}(t)=P_{r}$. Then, the energy replenished during any time interval $\left[t_{1}, t_{2}\right]$ is given by Formula 2 .

$$
g\left(t_{1}, t_{2}\right)=\left(t_{2}-t_{1}\right) \times P_{r}
$$

Below, we use $P_{r}$ instead of $P_{r}(t)$ to denote the replenishment function and we suppose that $P_{r} \leq \mathcal{C}$ to avoid energy loss. The replenishment process in energy harvesting systems is usually slower than the dissipation, for this reason we suppose that tasks consume more energy than the one which is replenished during executions, i.e. $\forall i, E_{i} \geq C_{i} \times P_{r}$.

We define the processor utilization of $\tau_{i}$ as $u_{i}^{p}=C_{i} / T_{i}$ and its energy utilization as $u_{i}^{e}=$ $E_{i} /\left(T_{i} \times P_{r}\right)$. The total utilization of the system is the sum of all the tasks utilization, i.e. $U^{p}=\sum_{i=1}^{n} u_{i}^{p}$ and $U^{e}=\sum_{i=1}^{n} u_{i}^{e}$.

In the considered model, the system has to respect all deadlines and energy constraints, namely tasks energy cost and battery capacity. The system executes and consumes energy when it is available and only replenishes it when it is not. The battery energy level never exceeds its threshold $E_{\max }$ nor fall below $E_{\min }$. Thus, a taskset is feasible if and only if there is a schedule where all the deadlines are met and the battery level never fall below $E_{\text {min }}$.

Figure 1 recapitulates these descriptions.

\section{Theoretical Study of $P F P_{A S A P}$}

\subsection{As Soon As Possible Preemptive Fixed-Priority Algorithm}

In [7, a scheduling algorithm for energy harvesting systems was introduced. This algorithm is a fixed-priority one which takes into account the tasks energy cost and the battery capacity during scheduling operations. Tasks are executed according to their priority, furthermore, whenever there is not enough energy to execute, jobs execution can be suspended to replenish energy for a fixed amount of time $x$. The authors performed tests by varying the $x$ parameter from $x=4$ to $x=100$ 


\begin{tabular}{|c|c|c|c|c|c|}
\hline- & $C_{i}$ & $E_{i}$ & $T_{i}$ & $D_{i}$ & $P_{i}$ \\
\hline$\tau_{1}$ & 4 & 216 & 32 & 16 & 1 \\
\hline$\tau_{2}$ & 1 & 48 & 48 & 32 & 2 \\
\hline$\tau_{3}$ & 1 & 16 & 48 & 22 & 3 \\
\hline$\tau_{4}$ & 3 & 186 & 40 & 32 & 4 \\
\hline
\end{tabular}

(a) Taskset $\Gamma_{1}$

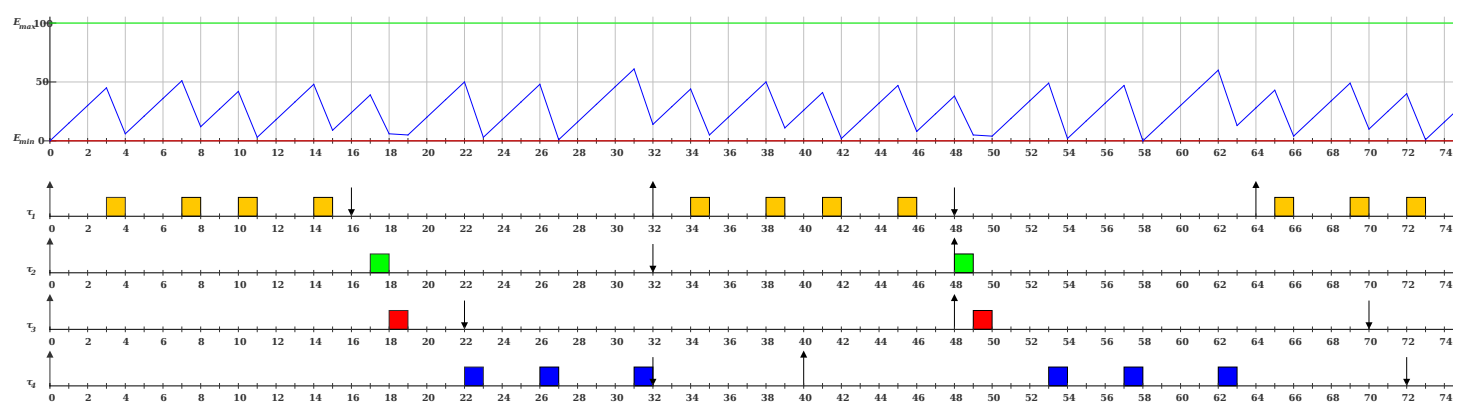

(b) $P F P_{A S A P}$ time chart for taskset $\Gamma_{1}$

Figure 2: A PFP $P_{A S A P}$ schedule

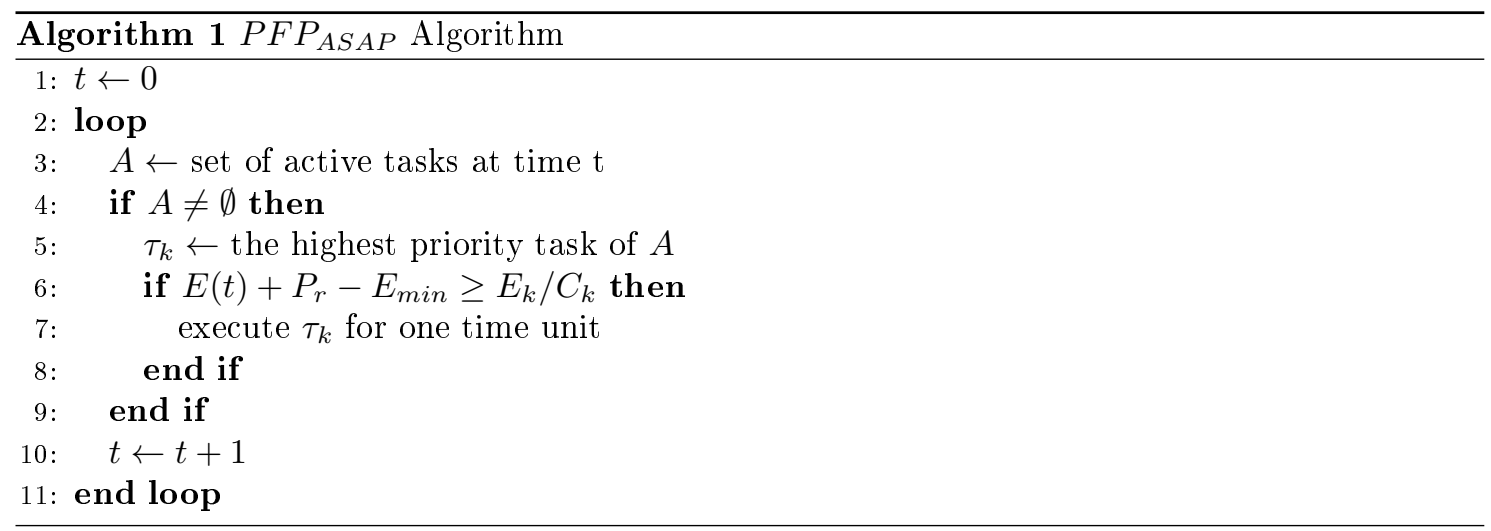


and the best value for their tasksets sample was 6 . However, they did not evaluate the algorithm for $x=1$.

In this section we study a special case of this algorithm, one where $x=1$ that we call As Soon As Possible Preemptive Fixed-Priority Algorithm (PFP $A S A P)$. Algorithm 1 shows how PF $P_{A S A P}$ takes decisions at time $t$. It schedules jobs as soon as possible when there is enough energy to execute one time unit, otherwise, it suspends tasks executions to replenish the battery. The replenishment periods are as long as needed for the execution of one time unit.

Figure 2(b) illustrates an PFP $P_{A S A P}$ schedule of the taskset $\Gamma_{1}$ described in Table 2(a) in the time interval [0,72]. In this example we have $E_{\max }=100, E_{\min }=0$ and $P_{r}=15$. At time $t=0$ the battery is empty, therefore, task $\tau_{1}$ cannot be executed. The battery is replenished until time $t=3$, i.e. until there is enough energy to execute one time unit of $\tau_{1}$. Then, the algorithm follows the same scheduling rules for the rest of the schedule.

Below, we will first address the PFP $P_{A S A P}$ worst case scenario, then we will discuss its optimality and finally, we will build a necessary and sufficient feasibility condition for the scheduling problem.

\subsection{Worst Case Scenario}

The aim of this section is to characterize the worst case scenario that a taskset can encounter during its execution. First, let us recall the notion of processor demand, then we will extend it to include task energy consumption.

Definition 1. The processor demand of the $i^{\text {th }}$ priority level at time $t$ denoted as $w p_{i}(t)$, is the amount of time necessary to execute jobs of priority levels $1, \ldots, i-1, i$ requested in the interval of time $[0, t]$. It can be obtained by formula 3 .

$$
w p_{i}(t)=\sum_{j \leq i}\left\lceil\frac{t-O_{j}}{T_{j}}\right\rceil \times C_{j}
$$

Now we introduce the notion of replenishment demand.

Definition 2. The replenishment demand of the $i^{\text {th }}$ priority level at instant $t$ denoted $w e_{i}(t)$, is the amount of energy to be replenished to execute jobs of priority levels $1, \ldots, i-1, i$ requested in the interval of time $[0, t]$. It can be calculated by formula 4

$$
w e_{i}(t)=\sum_{j \leq i}\left\lceil\frac{t-O_{j}}{T_{j}}\right\rceil \times E_{j}-E(0)
$$

The intuition of formula 4 is derived from the notion of processor demand. It represents the sum of the cost of energy of all the jobs of priority equal or higher than $i$ requested during the time interval $[0, t]$. Then, we remove the initial battery level $E(0)$ to fit the exact amount of energy to be replenished.

Definition 3. The time demand of the $i^{\text {th }}$ priority level at instant $t$ denoted as $w_{i}(t)$, is the minimum amount of time necessary to satisfy both of the replenishment and processor demand in the time interval $[0, t]$. This can be calculated by formula 5 .

$$
w_{i}(t)=\max \left(\left\lceil\frac{w e_{i}(t)}{P_{r}}\right\rceil, w p_{i}(t)\right)
$$

Definition 4. The response time of the first job of $\tau_{i}$ according to PFP $P_{A S A P}$ denoted as $R_{i}$ is the execution termination date of $i^{\text {th }}$ priority level minus $O_{i}$. The termination date of the first job of $\tau_{i}$ denoted as $t_{f}$ is the smallest solution of the system of equations 6 .

$$
\left\{\begin{array}{l}
w_{i}\left(t_{f}\right)=\max \left(\left\lceil\frac{w e_{i}\left(t_{f}\right)}{P_{r}}\right\rceil, w p_{i}\left(t_{f}\right)\right) \\
w_{i}\left(t_{f}\right)=t_{f} \\
w_{i}\left(t_{f}\right)>O_{i}
\end{array}\right.
$$



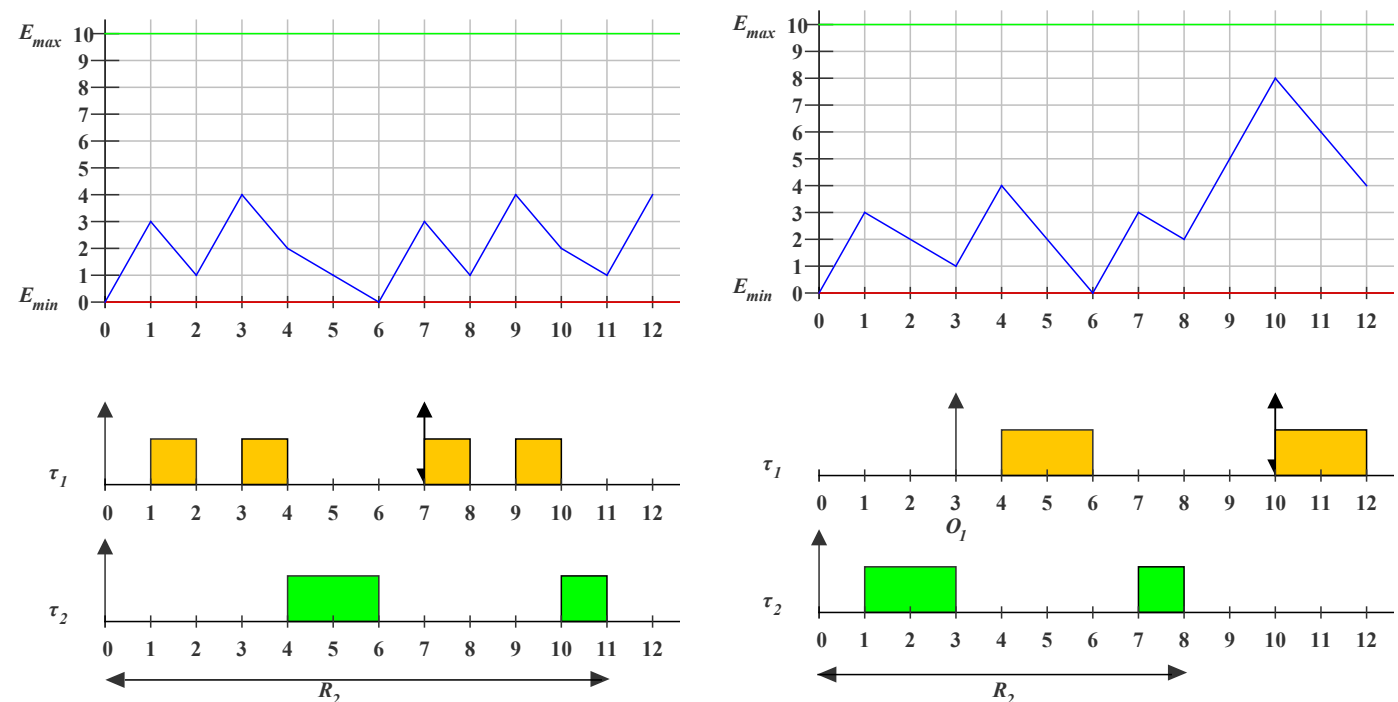

(a) Synchronous activation

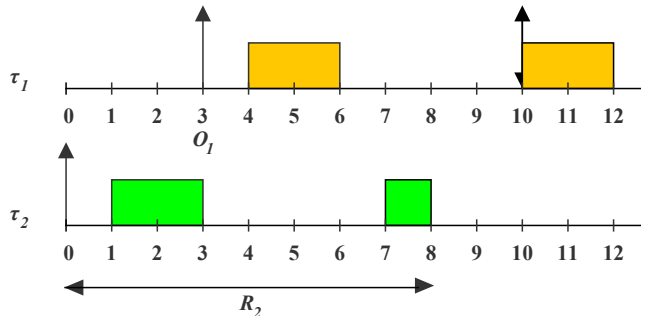

(b) Higher priority tasks are activated later
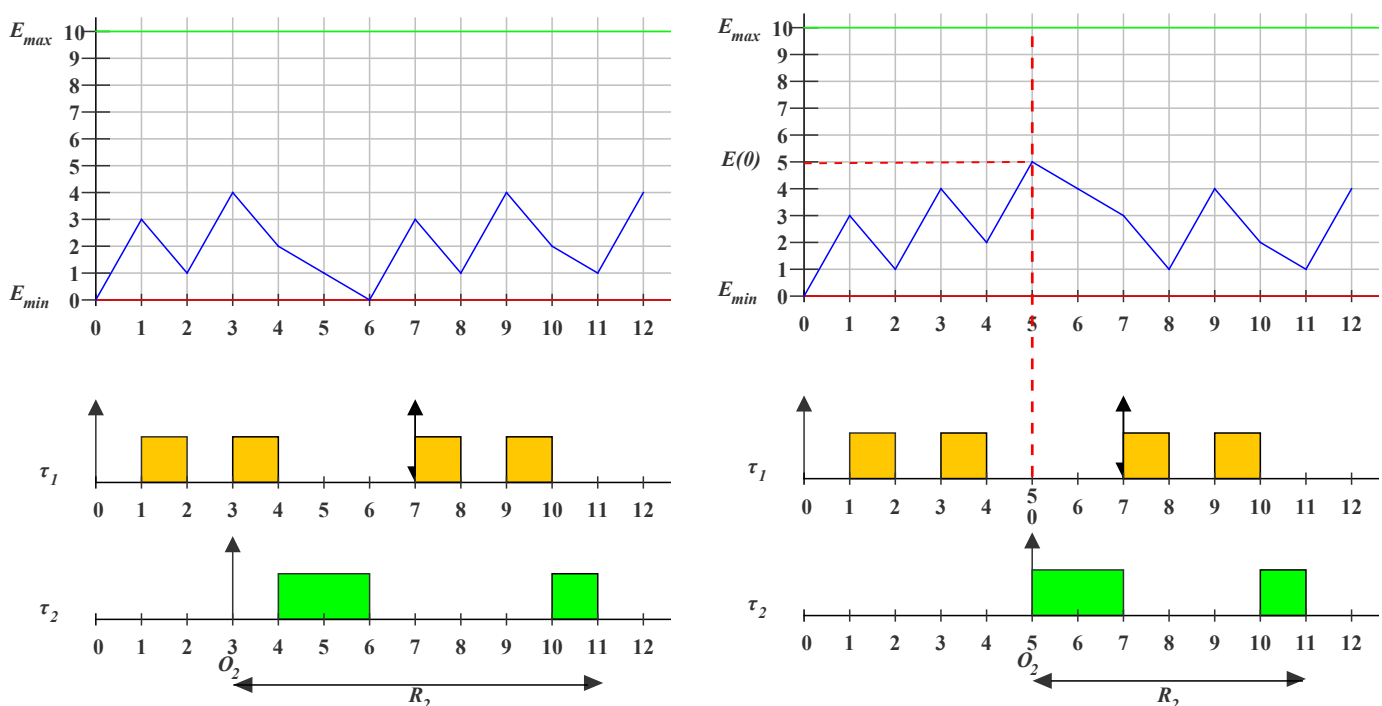

(c) Higher priority tasks are activated earlier

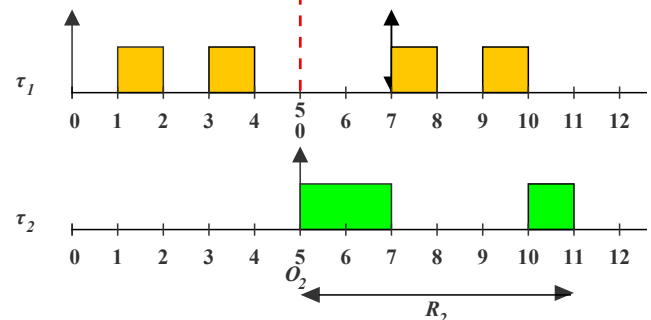

(d) Higher priority tasks are activated earlier with modified offsets

Figure 3: Response time in different activation scenarios 
Now, we can use these definitions to characterize the worst case scenario which is expected to be the synchronous activation of all the tasks when the battery is at its minimum level. This intuition is justified by the comparison of all possible activation scenarios as shown in Figure 3 .

Figure 3(a) illustrates the case where all the tasks are requested simultaneously. If at least one higher priority task is requested later, the response time of lower priority tasks decreases as illustrated in Figure 3(b). Then, if higher priority tasks are requested earlier, the response time of lower priority tasks cannot be longer than the one in the synchronous scenario as shown in Figures $3(\mathrm{c})$ and $3(\mathrm{~d})$.

Thus, we propose Theorem 1.

Theorem 1. Let $\Gamma$ denote a non concrete taskset composed of $n$ priority-ordered tasks with constraint or implicit deadlines. The PFP $P_{A S A P}$ worst case scenario for any task of $\Gamma$ occurs whenever this task is requested simultaneously with requests of all higher priority tasks and the battery is at the minimum level $E_{\text {min }}$.

Proof. We will compare the jobs response times in the scenario of the theorem with all other possible ones. As mentioned earlier, the response time of a job is equal to its termination date minus its offset. The main key of the proof is to argue with termination dates and offsets by comparing their possible values in different cases of activation scenario.

Let $\left\{\tau_{1}, \tau_{2}, \ldots, \tau_{n}\right\}$ be a set of $n$ priority-ordered tasks where $\tau_{n}$ is the task with the lowest priority. Let $S_{i}^{s}$ denote the scenario where task $\tau_{i}$ and all higher priority tasks are requested simultaneously at the lower battery level $E_{\min }$. The worst case scenario for a task $\tau_{i}$ is the one which maximizes its response time, i.e. the scenario which maximizes the termination date of the first job of the $i^{\text {th }}$ priority level.

If $S_{i}^{s}$ is not the worst scenario, there must be an other one leading to a greater response time for the $i^{\text {th }}$ priority level.

Firstly, we consider the scenario where $E(0)>E_{\min }$. In this case there is some amount of energy available at time $t=0$. Therefore, the system needs less replenishment demand than the scenario where $E(0)=E_{m i n}$, and $P F P_{A S A P}$ introduces shorter or equal replenishment periods and leads to shorter response time for all the tasks. This is in contradictory with our hypothesis, thus, such a scenario cannot lead to longer response times.

Secondly, we consider the scenario with different offsets. Let us denote $S_{i}^{a}$ as the scenario where $E(0)=E_{\min }=0$ and all tasks have different offsets. Let $t_{s}$ denote the termination date of the first job of task $\tau_{i}$ in the synchronous scenario $S_{i}^{s}$ and let $t_{a}$ denote the termination date of the same job in the asynchronous scenario $S_{i}^{a}$. Scenario $S_{i}^{a}$ is worse than scenario $S_{i}^{s}$ implies that $t_{a}>t_{s}$.

We know that

$$
t_{s}=w_{i}^{s}\left(t_{s}\right)=\max \left(\left\lceil\frac{w e_{i}\left(t_{s}\right)}{P_{r}}\right\rceil, w p_{i}\left(t_{s}\right)\right)
$$

and $\left[\frac{w e_{i}\left(t_{s}\right)}{P_{r}}\right] \geq w p_{i}\left(t_{s}\right)$ because in our model $E(0)=0$ and $\forall i, E_{i} \geq C_{i} \times P_{r}$. This reveals the fact that in the considered model, we must have replenishment periods which increase job response time. Then,

$$
t_{s}=w_{i}^{s}\left(t_{s}\right)=\left\lceil\frac{\sum_{j \leq i}\left\lceil\frac{t_{s}}{T_{j}}\right\rceil \times E_{j}}{P_{r}}\right\rceil
$$

Similarly,

$$
t_{a}=w_{i}^{a}\left(t_{a}\right)=\left\lceil\frac{\sum_{j \leq i}\left\lceil\frac{t_{a}-O_{j}}{T_{j}}\right\rceil \times E_{j}}{P_{r}}\right\rceil
$$


Knowing that $w_{i}^{a}(t)$ is strictly increasing in the interval $\left[0, t_{a}\right]$ and $t_{a}=w_{i}^{a}\left(t_{a}\right)$, we obtain

$$
t_{s}<t_{a} \Rightarrow t_{s}<w_{i}^{a}\left(t_{s}\right)
$$

By replacing $t_{s}$ with $w_{i}^{s}\left(t_{s}\right)$ we obtain

$$
\left\lceil\frac{\sum_{j \leq i}\left\lceil\frac{t_{s}}{T_{j}}\right\rceil \times E_{j}}{P_{r}}\right\rceil<\left\lceil\frac{\sum_{j \leq i}\left\lceil\frac{t_{s}-O_{j}}{T_{j}}\right\rceil \times E_{j}}{P_{r}}\right\rceil
$$

Finally, we have

$$
\sum_{j \leq i}\left\lceil\frac{t_{s}}{T_{j}}\right\rceil \times E_{j}<\sum_{j \leq i}\left\lceil\frac{t_{s}-O_{j}}{T_{j}}\right\rceil \times E_{j}
$$

We know that $t_{s} \geq t_{s}-O_{j}$ because $O_{j} \geq 0$. Therefore

$$
\sum_{j \leq i}\left\lceil\frac{t_{s}}{P_{r}}\right\rceil \times E_{j} \geq \sum_{j \leq i}\left\lceil\frac{t_{s}-O_{j}}{P_{r}}\right\rceil \times E_{j}
$$

Inequality 12 is in contradiction with inequality 13 . Thus, we prove that $t_{s} \geq t_{a}$. Knowing that $R_{i}=t_{f}-O_{i}$, we also have $R_{i}^{s} \geq R_{i}^{a}$ because $t_{s}-0 \geq t_{a}-O_{i}$.

\subsection{Optimality}

Theorem 2. PFP $P_{A S A P}$ is optimal for the scheduling problem of non concrete tasksets with constrained or implicit deadlines.

Proof. Let $\Gamma$ denote a non concrete taskset. We suppose that $\Gamma$ is feasible using a fixed-priority assignment, but not schedulable with $P F P_{A S A P}$ using the same priority assignment. This means that at least one task denoted as $\tau_{k}$ misses its deadline during the first instance of the worst case scenario (see Theorem 1). Indeed, it is sufficient to consider only the first job because deadlines are constrained or implicit. According to PFPASAP rules, a deadline miss occurs in the worst case scenario for the $k^{t h}$ priority level only if the energy needed to execute priority levels higher or equal to $k$ is greater than the energy that can be replenished from $t=0$ to the first deadline of $\tau_{k}$, Inequality 14 summarizes that.

$$
D_{k} \times P_{r}<\sum_{j \leq k}\left\lceil\frac{D_{k}}{T_{j}}\right\rceil \times E_{j}
$$

If $P F P_{A S A P}$ is not optimal, there must be an other fixed-priority schedule for $\Gamma$ that makes it feasible. Let us suppose that such a schedule exists. This implies that there exists at least one task which is executed even if the energy is not sufficient. This is impossible because the system cannot execute without energy, therefore such a schedule cannot exist. Then we prove that PFP $P_{A S A P}$ is optimal for non concrete fixed-priority tasksets with constrained or implicit deadlines.

\section{Discussion}

The optimality of $P F P_{A S A P}$ relies on the hypothesis fixed in Section 3 mainly the ones about task consumption and replenishment functions. If we relax some of them $P F P_{A S A P}$ may lose its optimality. 
Up to now, we have only dealt with linear consumption. If we model consumption as a non linear function, $P F P_{A S A P}$ may not be able to estimate the energy to be replenished to execute exactly one time unit and can lose its optimality.

Tasks consuming less energy than the replenished one are not considered in our model. Including this kind of tasks makes the priority ordering relevant in response time computation and makes the proof we provided insufficient.

In a more realistic model, the replenishment function is not constant. Therefore Equation 14 is no more valid. Thus, we cannot conclude about $P F P_{A S A P}$ optimality. Finally, we have counter examples that prove the non-optimality of $P F P_{A S A P}$ for concrete tasksets.

\subsection{Feasibility Condition}

A simple way to build a necessary and sufficient feasibility condition for non concrete tasksets is to check if the given taskset is schedulable with $P F P_{A S A P}$ in the worst case scenario, i.e. check if the first job of each task meets its deadline when it is requested simultaneously with the higher priority while the battery is at its minimum level. It consists in computing the worst response time according to $P F P_{A S A P}$ rules for each task and comparing it to its first deadline. Algorithm 2 explains how to do this.

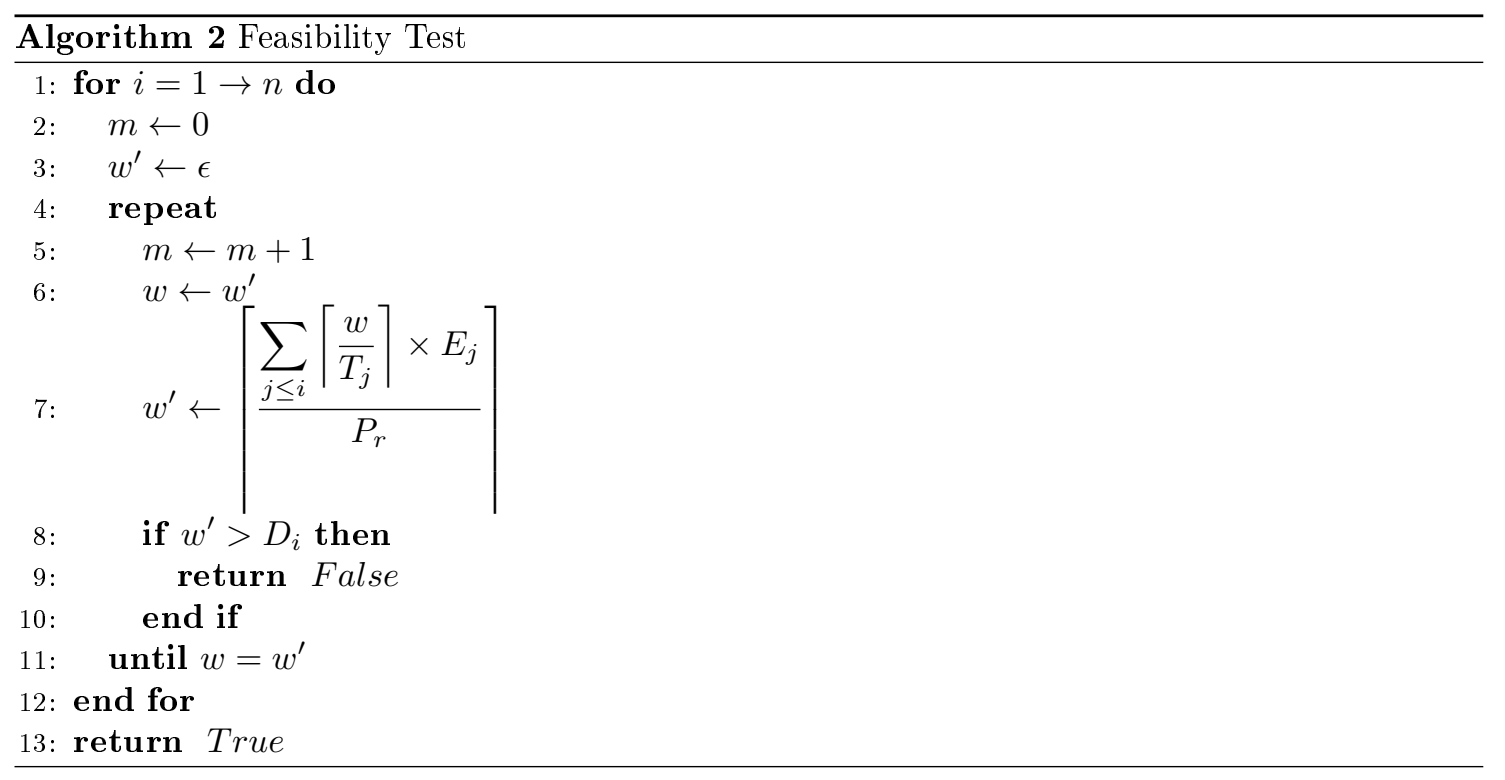

The complexity of Algorithm 2 is $O(m \times n)$ where $m$ is the number of iterations and $n$ is the number of tasks. We note that the number of iterations $m$ depends on the periods and deadlines of the tasks (see line 8) and is bounded by $\max _{\forall i}\left(D_{i}\right)$. Thus, the complexity of Algorithm 2 is pseudo-polynomial. We can reduce this complexity by computing estimations for response times rather than the exact values. However, the feasibility test we propose will not be longer necessary but will remain sufficient.

\subsection{Battery Capacity}

The design of a system with an arbitrary battery capacity may lead to an overestimated $\mathcal{C}$, which can be very costly (space, weight, money). Finding the lowest battery capacity value is a very important issue.

Given a feasible taskset with $\mathcal{C}=\infty$, the minimum battery capacity issue in harvesting systems is to find the smallest value of $\mathcal{C}$ denoted as $\mathcal{C}^{\text {min }}$ that keeps the taskset feasible when we launch the system at the minimum battery level. 


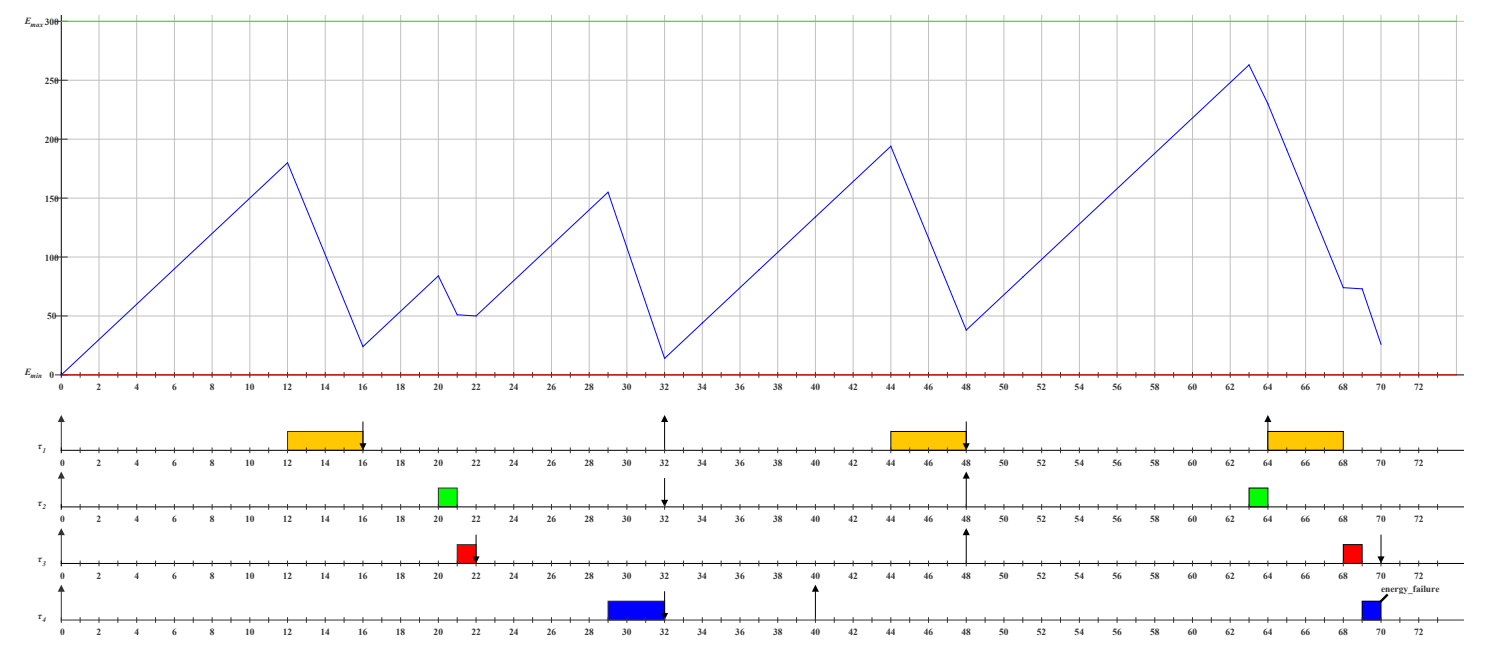

Figure 4: $P F P_{A L A P}$ schedule for taskset $\Gamma_{1}$

The exact value of $\mathcal{C}^{\text {min }}$ is difficult to estimate because it depends on the environmental characteristics and the used scheduling algorithm. We can solve the problem by bounding the $\mathcal{C}^{\text {min }}$ value, however, in the case of PFP $A S A P$ algorithm, we can compute the exact value.

Algorithm PFP AS AP replenishes the minimum amount of energy needed for only one execution time unit, in this case the minimum battery capacity needed to keep the taskset feasible is the maximum amount of energy that can be consumed during one time unit, i.e. the maximum instantaneous consumption. In our model all the tasks consume energy linearly. Therefore, the minimum battery capacity that keeps the taskset feasible is $\mathcal{C}^{\text {min }}=\max _{\forall i}\left(E_{i} / C_{i}\right)$ in the general case, and $\mathcal{C}^{\text {min }}=\max _{\forall i}\left(E_{i} / C_{i}\right)-P_{r}$ with the constant replenishment function hypothesis.

If we relax the hypothesis related to the consumption model, a task can at worst consume all its energy cost $E_{i}$ during the first execution time unit. In this case the minimum battery capacity needed to execute one time unit is the maximum of tasks cost, i.e. $\mathcal{C}^{\text {min }}=\max _{\forall i}\left(E_{i}\right)$.

In both cases, the battery capacity cannot be lower because if we deal smaller battery capacity, the system will never be able to execute one time unit and will never have enough energy to execute. Therefore, no other algorithm can run with smaller battery capacity.

\section{Performance Evaluation}

We proved that $P F P_{A S A P}$ is optimal for non concrete tasksets. In this section we study the behavior of $P F P_{A S A P}$ and we compare it to other algorithms by simulations and analyze its performance.

Some scheduling algorithms and heuristics were proposed in [7, 5]. We selected $P F P_{S T}$ which is not optimal but has the lowest failure rate according to the experiment performed in [7]. We also selected the $P F P_{A L A P}$ algorithm because it can be used to implement a sufficient feasibility condition [5]. In this section, we compare these two algorithms with $P F P_{A S A P}$, then we analyze their performance.

\subsection{Competitors}

- $P F P_{A L A P}$ : is a fixed-priority scheduling algorithm that postpones all jobs execution as late as possible, i.e. it introduces idle-periods that consume all available slack-time to charge energy before each job execution. The $P F P_{A L A P}$ algorithm may lose energy if $E_{\max }$ is 
reached before the end of an idle period. Figure 4 illustrates an $P F P_{A L A P}$ schedule at time interval $[40,80]$ of the taskset $\Gamma_{1}$ described in Table $2(\mathrm{a})$

- $P F P_{S T}$ : is a fixed-priority scheduling heuristic that executes tasks as soon as possible when there is energy available in the battery, and replenishes it when it is not. The replenishment periods are as long as available slack-time. Tasks execution is resumed whenever $E_{\max }$ is reached.

\subsection{Simulation}

In this section, we describe the configuration of the experiments, namely the simulation tool, the input data, the parameters and the set assumptions.

\subsubsection{Simulation Tool}

To perform such an experiment, we need a simulation tool able to run large scale simulations on various data and algorithms. We used YARTISS, a simulation tool presented in [6. It provides a simulation framework able to run the simulation of a large set of tasksets on different energy parameters and according to different scheduling algorithms simultaneously. It can also provide statistics about the performed simulations like the failure rate, the preemption rate or the average battery level during the simulations and many other metrics. The tool is available on-line in [1].

\subsubsection{Input Data}

For these simulations we used an adapted version of the UUniFast-Discard algorithm [4 coupled with a limitation of hyper-period technique [13 to generate tasksets. The generated tasksets respect the following hypotheses:

- all tasksets are time feasible,

- time and energy are discretized, this means that they are integers and all scheduling operations are performed before or after one time unit,

- the charging function $P_{r}$ is constant, i.e. a constant amount of energy is added to the battery level in every time unit,

- tasks consume energy linearly, i.e. a task consumes $E_{i} / C_{i}$ energy units for each execution time unit.

In order to represent most of the possible tasksets, we generate them according to their processor and energy utilizations, i.e. $U^{p}$ and $U^{e}$. We vary $U^{p}$ and $U^{e}$ in the interval $[0.2,1]$ to obtain a couple of $\left(U^{p}, U^{e}\right)$ for each 0.05 unit of $U^{p}$ and $U^{e}$. Then, we obtain 350 distinct tasksets for each couple $\left(U^{p}, U^{e}\right)$.

In this paper we restricted the study to non concrete tasksets. Therefore, all tasksets are simulated in the worst case scenario. The data used for our simulations are available online in [1].

\subsubsection{Simulation Description}

In order to evaluate the behavior of the compared algorithms, we vary some parameters, namely the battery capacity $\mathcal{C}$ and the number of tasks per taskset. Firstly, we vary $\mathcal{C}$ in six energy scenarios to analyze its effect on the failure rate. Secondly, we vary the number of tasks per taskset in several distinct simulations to observe the evolution of the scheduling overhead of each algorithm.

We set the remaining parameters to the same values that we used for tasksets generation in order to fit the considered assumptions. For these experiments, we set these parameters as follows, $P_{r}=15, E_{\min }=0$. The simulations are executed for 3000 discretized time units. Furthermore, 
tasksets are run for more time than one hyper-period that is bounded by 2500 time units. Thus, if a taskset does not miss any deadline during the simulation time, the taskset is said to be feasible. We use Deadline Monotonic policy (DM) to assign priorities because of its optimality for the classical scheduling problem. However, DM policy loses its optimality when we integrate energy constraints (tasks consumption, battery capacity) but according to preliminary experiments, it is still dominating Rate Monotonic.

Several statistical metrics are computed during simulations. These metrics give information about algorithms behavior. For our experiments we selected the following metrics: failure rate, Preemption count, Average overhead, Average idle-period, Average busy-period and Average energy level.

\subsubsection{Metrics Definition}

Failure Rate is the percentage of non feasible taksets among all the tested ones.

Preemption Count for one simulation, it represents the number of preemption events. A preemption event occurs when a job is stopped while it is still not finished. All of the events that occur at the same instant are considered once. Therefore, the number of possible events is bounded by the number of time units composing the simulation, i.e. the simulation duration. For several simulations, this metric is computed only for feasible tasksets and represents the ratio of the average number of preemptions relative to all possible events.

Average Overhead it is the amount of time spent while handling a scheduling event. For one simulation, this metric represents the average overhead of all of the scheduling events. Its exact value is difficult to compute. We simply calculate an estimate by distributing the real simulation time (in milliseconds) upon the number of scheduling events. The simulation tool that we use is event-based. Therefore, only the events handling consume processor time. Thus, it gives us an acceptable estimation of the average overhead.

Average Idle-Period represents the average duration of periods when the processor is idle. It includes battery replenishment and slack-time periods. For several simulations, we compute the ratio of the average idle-period duration relative to the simulation duration.

Average Busy-Period is the average duration of continuous processor activity. For several simulations, we compute the ratio of the average busy-period duration relative to the simulation duration.

Average Energy Level represents the average energy level of the battery or capacitor at any instant during the simulation. It is the average of the energy level of all scheduling events.

\subsubsection{Metrics Relevance}

Failure Rate the greater the failure rate, the lower the algorithm performance.

Preemption Count the greater the number of preemptions, the greater the context switch. This increases the overhead cost and decreases performance, which makes the algorithm unusable in practice.

Average Overhead the greater the average overhead, the greater the timing constraints violation risk. This makes the algorithms unusable in practice. 


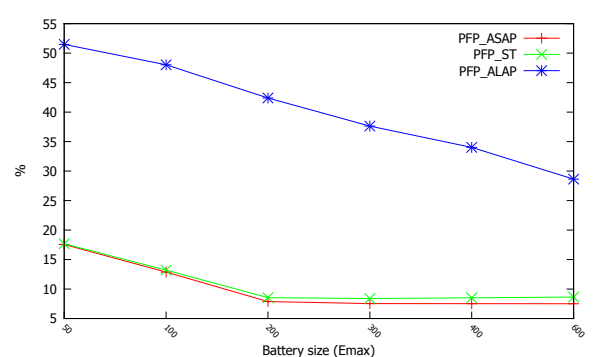

(a) Failure rate

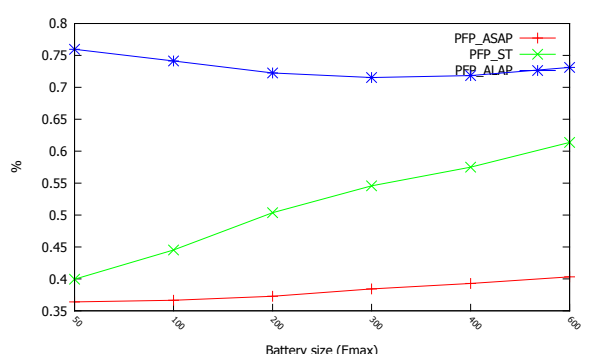

(c) Average idle period

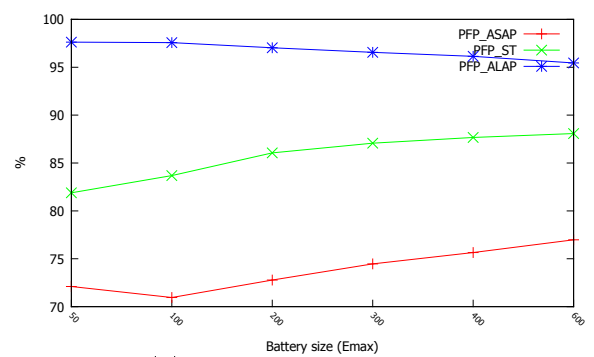

(e) Average energy level

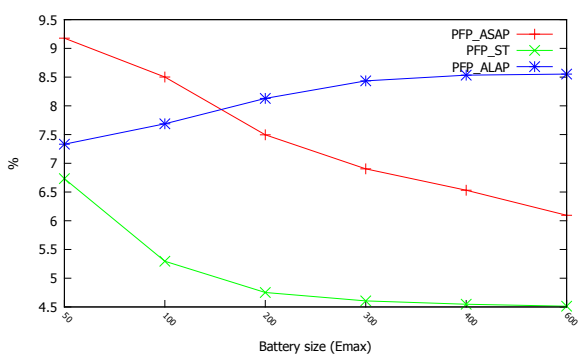

(b) Preemptions

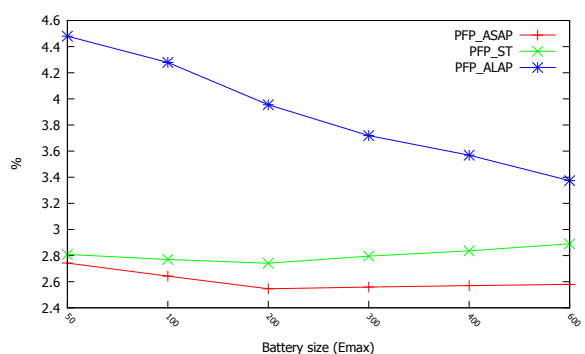

(d) Average busy period

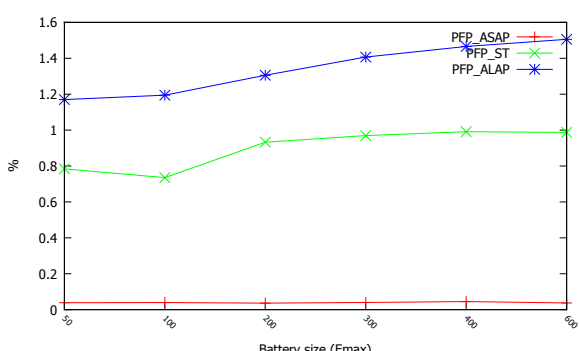

(f) Average overhead

Figure 5: Comparison between $P F P_{A L A P}, P F P_{S T}$ and $P F P_{A S A P}$

Average Idle-period and Average Busy-Period the relevance of these two metrics is closely linked to the number of preemptions. The longer the idle/busy periods, the lesser the number of preemptions and the higher the algorithm performance. Therefore, the longer the idle-periods, the higher the average energy level and the lesser energy-constrained the system.

Average Energy Level the best algorithm relative to this criterion is one which maximizes the average energy level. This means that the algorithm makes the system less energy constrained.

\subsection{Results Analysis}

\subsubsection{The Variation of $E_{\max }$}

Figure 5 presents the results of comparing the algorithms. In the following part, we analyze the effect of $E_{\max }$ varying on the performance of each algorithm for each metric:

Failure Rate The increase of $E_{\max }$ reduces the failure rate of all the evaluated algorithms. This result was expected because the more $E_{\max }$ is increased the less the system is energy-constrained. We also observe that $P F P_{A L A P}$ has the highest failure rate for all values of $E_{\max }$. Both of $P F P_{A S A P}$ and $P F P_{S T}$ have a lower failure while $P F P_{A S A P}$ demonstrates the lowest one. To explain why $P F P_{S T}$ fails to schedule some tasksets which are schedulable with $P F P_{A S A P}$ we 
have to examine its behavior closely. When the battery is down, $P F P_{S T}$ suspends the system as long as possible before the next execution while $P F P_{A S A P}$ suspends the system for only one time unit. In this case $P F P_{S T}$ may uselessly postpone executions and may accumulate an unbearable energy load for a future time. This can lead the system to replenish more time than the slack-time available and may lead to missing deadlines. PF $P_{A L A P}$ suffers from the same problem as $P F P_{S T}$ because it postpones execution as long as possible regardless of the battery level. When all jobs are postponed to a maximum and the system incurs a long execution period, it is impossible to introduce more replenishment periods. Therefore, deadline misses may occur. Figure 4 illustrates a deadline miss caused by delay similar to the one explained for $P F P_{S T}$. At time 63 a long busy period begins and the system has already consumed all the slack-time. The energy replenished during the former idle periods is not sufficient and the system runs out of energy. The PFPASAP algorithm allows to avoid this situation by starting jobs immediately.

As shown in Section 4 $P F P_{A S A P}$ is optimal for non concrete tasksets, the simulations confirm that. All of the tasksets that are feasible with $P F P_{A L A P}$ and $P F P_{S T}$ are still feasible with $P F P_{A S A P}$ but not the reverse. However, the difference in the failure rate between $P F P_{S T}$ and $P F P_{A S A P}$ is still negligible, the study of the rest of metrics may be crucial.

Preemption Rate The simulations show that increasing $E_{\max }$ helps to stabilize the number of preemptions. However $P F P_{A S A P}$ demonstrates a very high number of preemptions regardless $E_{\text {max }}$ values. By construction, $P F P_{A S A P}$ executes for one time unit then preempts tasks to check again if there is enough energy, while $P F P_{S T}$ consumes all the slack-time available to replenish energy and avoid preemptions due to a lack of energy. $P F P_{A L A P}$ does the same for each job activation.

Average Overhead We observe that for every value of $E_{\max }, P F P_{A L A P}$ and $P F P_{S T}$ have much higher average overhead than $P F P_{A S A P}$. This is due to the pseudo-polynomial complexity of the slack-time algorithm 9. $P F P_{A L A P}$ computes slack-time whenever a job is requested and $P F P_{S T}$ does the same only if there is not enough energy while $P F P_{A S A P}$ only needs to order the activated jobs.

Average Idle-Period and Busy-Period These two metrics are closely linked to the number of preemptions, the longer the idle or busy periods, the lower the number of preemptions. PF $P_{A S A P}$ maximizes the number of preemptions, therefore, it has shorter idle and busy periods. $P F P_{S T}$ consumes all available slack-time to replenish energy, then, executes tasks while the battery level is sufficient. This maximizes the duration of both of the idle and the busy periods.

Average Energy Level regardless $E_{\max }$ values, $P F P_{A L A P}$ has the highest average energy level and $P F P_{A S A P}$ has the lowest one. We expected this result because the $P F P_{S T}$ replenishes energy during long periods (idle periods) which increase the average battery level.

\subsubsection{Varying Taskset Cardinal}

The aim of this experiment is to study the effect of the taskset cardinal on the average overhead. We performed the simulations and the results confirmed our previous observations. Both of $P F P_{A L A P}$ and $P F P_{S T}$ have a very large overhead relative to $P F P_{A S A P}$. This is explained by the complexity of the slack-time computation algorithm.

Table 1 summarizes the performance of the evaluated algorithms.

$P F P_{A S A P}$ is optimal for non concrete tasksets and has the lowest failure rate compared to the other algorithms and need less battery capacity to operate. However, it increases the number of preemptions and context switches which can be considered to be a great disadvantage. PFP $P_{S T}$ is not optimal but simulations show that its failure rate is very close to that of $P F P_{A S A P}$. Furthermore, it maximizes the average energy level and reduces preemptions. The pseudo-polynomial 


\begin{tabular}{|c|c|c|c|}
\hline- & $P F P_{A L A P}$ & $P F P_{S T}$ & $P F P_{A S A P}$ \\
\hline Worst case scenario & - & - & synchronous activations \\
\hline Optimality & bad & bad & good \\
\hline Failure rate & bad & good & good \\
\hline Average overhead & bad & bad & bood \\
\hline preemptions & neutral & good & bad \\
\hline Average idle-period & neutral & good & bad \\
\hline Average busy-period & neutral & good & bad \\
\hline Average energy level & neutral & good & \\
\hline
\end{tabular}

Table 1: $P F P_{A L A P}$ vs $P F P_{S T}$ vs $P F P_{A S A P}$

complexity of stack-time calculation is the main drawback of $P F P_{S T}$, then, it cannot be used for systems with a large number of tasks. Concerning $P F P_{A L A P}$, in addition to its non-optimality, simulations demonstrate very bad performance for all metrics.

Finally, we can conclude that $P F P_{A S A P}$ is optimal but not applicable in practice because of its preemption rate. However, despite the great complexity of $P F P_{S T}$ and its non optimality, it is still the only algorithm that shows many advantages. It may be very interesting to study the possibility of significantly reducing the complexity of the slack-time computation by dealing with approximated values.

\section{Conclusion and Future Work}

This paper addresses the problem of fixed-priority scheduling for energy harvesting real-time systems. We proposed $P F P_{A S A P}$, an optimal algorithm for non concrete tasksets, then we built a necessary and sufficient feasibility condition based on $P F P_{A S A P}$ algorithm. We also proved that the worst case scenario for $P F P_{A S A P}$ occurs whenever all tasks are requested simultaneously while the battery is at its minimum level $E_{\text {min }}$. We performed large scale simulations to evaluate $P F P_{A S A P}$ performance and compared it to other algorithms. The experiment showed that the main drawback of $P F P_{A S A P}$ is its very large number of preemptions.

Moreover, the PFP $P_{A S A P}$ algorithm is only optimal for non concrete tasksets. As future work, we will explore other scheduling policies and look for more general feasibility condition. Firstly, we plan to study the possibility of finding a clairvoyant algorithm based on PFPASAP that can be optimal for both concrete and non concrete tasksets. Then, we will try to optimize the number of preemptions to make it usable in practice. Secondly, we will study the possibility of using our feasibility test to build an optimal priority assignment (OPA) based on Audsley's algorithm [3] and Davis' criteria [8. Finally, we will be interested in measuring the effect of the assumptions we set on both replenishment and task consumption functions, indeed, we will try to find the worst case of both consumption and replenishment models.

\section{References}

[1] D. Zhu, X. Qi, and H. Aydin, "Priority-Monotonic Energy Management for Real-Time Systems with Reliability Requirements," in Proceedings of the 25th International Conference on Computer Design, 2007.

[2] D. Zhu and H. Aydin, "Energy management for real-time embedded systems with reliability requirements," in Proceedings of the 2006 IEEE/ACM international conference on Computeraided design, 2006.

[3] A. Allavena and D. Mossé, "Scheduling of frame-based embedded systems with rechargeable batteries," in Workshop on Power Management for Real-Time and Embedded Systems (in conjunction with RTAS), 2001.

[4] C. Moser, D. Brunelli, L. Thiele, and L. Benini, "Real-time scheduling with regenerative energy," in Proceedings of 18th Euromicro Conference on Real-Time Systems, 2006. 
[5] R. Jayaseelan and T. Mitra, "Estimating the Worst-Case Energy Consumption of Embedded Software," in Proceedings of the 12th IEEE Real-Time and Embedded Technology and Applications Symposium, 2006.

[6] H. EL Ghor, M. Chetto, and R. H. Chehade, "A real-time scheduling framework for embedded systems with environmental energy harvesting," Computers and Electrical Engineering, vol. 37, pp. 498-510, July 2011. [Online]. Available: http://dx.doi.org/10.1016/ j.compeleceng.2011.05.003

[7] M. Chetto, D. Masson, and S. Midonnet, "Fixed Priority Scheduling Strategies for Ambient Energy-Harvesting Embedded systems," in Proceedings of IEEE/ACM International Conference on Green Computing and Communications, 2011.

[8] J. P. Lehoczky and S. Ramos-Thuel, "An optimal algorithm for scheduling soft-aperiodic tasks fixed priority preemptive systems," in Proceedings of the 13th IEEE Real-Time Systems Symposium, 1992.

[9] Y. Chandarli, Y. Abdeddaïm, and D. Masson, "The Fixed Priority Scheduling Problem for Energy Harvesting Real-Time Systems," in Proceedings of WIP RTCSA, 2012.

[10] Y. Chandarli, F. Fauberteau, D. Masson, S. Midonnet, and M. Qamhieh, "YARTISS : A Tool to Visualize, Test, Compare and Evaluate Real-Time Scheduling Algorithms," in Proceedings of 3rd International Workshop on Analysis Tools and Methodologies for Embedded and Realtime Systems, 2012.

[11] "YARTISS," 2012. [Online]. Available: http://yartiss.univ-mlv.fr/

[12] E. Bini and G. C. Buttazzo, "Measuring the Performance of Schedulability Tests," Real-Time Systems, vol. 30, no. 1-2, pp. 129-154, May 2005.

[13] C. Macq and J. Goossens, "Limitation of the hyper-period in real-time periodic task set generation," in Proceedings of the 9th international conference on real-time systems, 2001.

[14] R. I. Davis, K. Tindell, and A. Burns, "Scheduling slack time in fixed priority pre-emptive systems," in Proceedings of the 14th IEEE Real-Time Systems Symposium, 1993.

[15] N. Audsley, Optimal Priority Assignment and Feasibility of Static Priority Tasks with Arbitrary Start Times, ser. Technical report. University of York, Department of Computer Science, 1991. [Online]. Available: http://books.google.fr/books?id=ooZkGwAACAAJ

[16] R. I. Davis and A. Burns, "Priority assignment for global fixed priority pre-emptive scheduling in multiprocessor real-time systems," in Proceedings of the 2009 30th IEEE Real-Time Systems Symposium, 2009. 